\title{
Wafer-Scale Leaning Silver Nanopillars for Molecular Detection at Ultra-Low Concentrations
}

Wu, Kaiyu; Rindzevicius, Tomas; Schmidt, Michael Stenbæk; Mogensen, Klaus Bo; Hakonen, Aron; Boisen, Anja

\section{Published in:}

The Journal of Physical Chemistry Part C

Link to article, DOI:

10.1021/jp510073y

Publication date:

2015

Document Version

Peer reviewed version

Link back to DTU Orbit

Citation (APA):

Wu, K., Rindzevicius, T., Schmidt, M. S., Mogensen, K. B., Hakonen, A., \& Boisen, A. (2015). Wafer-Scale Leaning Silver Nanopillars for Molecular Detection at Ultra-Low Concentrations. The Journal of Physical Chemistry Part C, 119(4), 2053-2062. https://doi.org/10.1021/jp510073y

\section{General rights}

Copyright and moral rights for the publications made accessible in the public portal are retained by the authors and/or other copyright owners and it is a condition of accessing publications that users recognise and abide by the legal requirements associated with these rights.

- Users may download and print one copy of any publication from the public portal for the purpose of private study or research.

- You may not further distribute the material or use it for any profit-making activity or commercial gain

- You may freely distribute the URL identifying the publication in the public portal 


\section{Wafer-Scale Leaning Silver Nanopillars for}

\section{Molecular Detection at Ultra-Low Concentrations}

Kaiyu Wu,,$^{\dagger}$ Tomas Rindzevicius, ${ }^{\dagger} *$ Michael Stenbcek Schmidt, ${ }^{\dagger}$ Klaus Bo Mogensen, ${ }^{\dagger}$ Aron

Hakonen, ${ }^{+}$and Anja Boisen ${ }^{\dagger}$

$\dagger$ Technical University of Denmark

Department of Micro- and Nanotechnology

Ørsteds Plads, Building 345 east, 2800 Kgs. Lyngby

\$Chalmers University of Technology

Department of Applied Physics

Gothenburg, 41296 Sweden

*Technical University of Denmark

Department of Micro- and Nanotechnology

Ørsteds Plads, Building 345 east, 2800 Kgs. Lyngby

Ph.: +4545255728

E-mail: trin@nanotech.dtu.dk 


\section{ABSTRACT}

Wafer-scale surface-enhanced Raman scattering (SERS) substrates fabricated using maskless lithography are important for scalable production targets. Large-area, leaning silver-capped silicon nanopillar (Ag NP) structures suitable for SERS molecular detection at extremely low analyte concentrations are investigated. Theoretical results show that isolated Ag NPs essentially support two localized surface plasmon (LSP) modes. The most prominent LSP resonance is observed in the near-infrared region $(\sim 800 \mathrm{~nm})$ and can be tuned by changing the diameter of the silicon nanopillars ( $\mathrm{Si}$ NPs). The corresponding electric field distribution maps indicate that the maximum E-field enhancement is found at the Ag cavity, i.e. the bottom part of the Ag cap. We argue that the plasmon coupling between the resonant Ag cap cavities contributes most to the enhancement of the Raman signal. We experimentally evaluate these findings and show that by exposing Si NPs to an $\mathrm{O}_{2}$-plasma the average Ag NP cluster size, and thus the overall inter-pillar coupling, can be systematically reduced. We show that deposition of $\mathrm{Cr}$ adhesion layers on Si NPs $(>3 \mathrm{~nm})$ introduce plasmon coupling loss to the Ag NP LSP cavity mode that significantly reduces the SERS intensity. Results also show that short exposures to the $\mathrm{O}_{2}$-plasma and the use of 1-3 $\mathrm{nm} \mathrm{Cr}$ adhesion layers are advantageous for reducing the signal background noise from Ag NPs. In addition, influence of the Ag NP height and Ag metal thickness on SERS intensities is investigated and optimal fabrication process parameters are evaluated. Finally, the SERS spectrum from $100 \mathrm{pM}$ of trans-1,2-bis (4-pyridyl) ethylene (BPE) is recorded showing distinct characteristic Raman vibrational modes. The calculated enhancement factor is of the order of $10^{8}$, and the SERS signal intensity exhibits a standard deviation of around 14\% (660 data points) across a 5x5 $\mathrm{mm}^{2}$ surface area.

Keywords: mask-less reactive ion etching, surface-enhanced Raman spectroscopy, hot spots, plasmonics 


\section{INTRODUCTION}

Surface Enhanced Raman Scattering (SERS) $)^{1-3}$ is a well-established spectroscopic technique for chemical and biological sensing. Noble metal nanostructures support localized surface plasmon resonances (LSPRs) ${ }^{4-6}$ that spatially confine the incident field and produce tremendous electromagnetic field enhancement spots, i.e. hot spots ${ }^{7}$. A broad variety of SERS substrates have been fabricated over the past decade, e.g. nanorods ${ }^{8}$, nanocubes $^{9}$, nanostars $^{10}$, various particle arrays ${ }^{11-16}$ and silver dendrites ${ }^{17}$. Recently electric field (E-field) enhancement factors (EF) above $10^{8}$ to even $10^{10}$ have been reported. ${ }^{1}$ Detection limits down to a single molecule level have also been reported. ${ }^{18-20}$ For a practical use, it is generally required that SERS substrates display (i) high and reproducible EF over large surface areas and (ii) a tunable LSPR wavelength over a broad spectral region. ${ }^{1}$ For applications that require massproduced SERS substrates, structures should also be robust, cost effective and involve few nanofabrication steps that are compatible with high volume manufacturing process flows. ${ }^{21}$

From the nanofabrication point of view, the majority of produced SERS substrates are either based on (i) metallic nanoparticles in colloidal suspensions, or (ii) roughened metallic surfaces. Various shapes of metallic nanoparticles in colloidal solutions showing high EFs with tunable LSPR wavelengths have been synthesized and their optical properties have been investigated both experimentally and theoretically., ${ }^{9,11-16}$ The main advantage of the SERS active colloidal suspensions is high EFs caused by the lightning-rod effect that is particularly pronounced in metal nanoparticles with sharp surface features. Decreasing the inter-particle separation, the LSPR coupling increases and the largest E-field enhancement values are usually obtained in the case of colloidal particle aggregates. In order to control the interparticle spacing, additional process steps are often needed. For instance, H. Wang et al. have reported a method to fabricate sub-10 nm gaps in highly ordered $\mathrm{Au}$ particle arrays functionalized with cetyltrimethylammonium bromide (CTAB). ${ }^{13}$ Sub-10 nm distance control between plasmonic nanoparticles can also be realized by bio-template assisted synthesis. ${ }^{15,16}$ 
Nanostructured surfaces can be tailored using a wide range of experimental approaches. ${ }^{1} \mathrm{~A}$ mask for a given nanostructure can initially be produced using lithographic techniques such as nanosphere $^{22}$ or e-beam lithography ${ }^{23}$. Then the final morphology is defined through metal deposition and resist lift-off processes that converts the mask into Raman active SERS substrates. ${ }^{22-26}$ Generally, such SERS substrates exhibit better nanostructure uniformities with more reproducible SERS signals across a larger surface area compared to e.g. particle colloids. One issue with this type of SERS substrates is that while the reproducibility of the SERS signal improves, the intensity usually decreases. ${ }^{27}$ Several methods have been developed to increase EFs by employing sharp metal particle features ${ }^{28,29}$ and by forming sub-10-nm gaps between the nanostructures ${ }^{30,31}$.

Vertically standing metal nanopillar (NP) structures are good candidates to fulfill both uniformity and high E-field enhancement requirements for reproducible molecular detection. Various NP arrays using e-beam lithography ${ }^{32}$, anodized aluminum oxide templates ${ }^{33,35}$, nanoimprinting $^{34}$, oxygen-plasma-stripping-of-photoresist technique ${ }^{36}$, ion milling ${ }^{37}$, interference lithography ${ }^{38,40}$, and coating of multi-walled carbon nanotubes ${ }^{39}$ have been reported. Recently, we have developed a new method to fabricate wafer scale Ag-capped Si nanopillar (Ag NP) SERS substrates utilizing maskless lithography. ${ }^{41,42}$ The fabrication method is particularly interesting due to its simplicity, low cost and high throughput that are necessary for commercial applications. These Ag NP substrates are capable of producing high E-field enhancement while the SERS signal uniformity remains relatively stable across a large (> $\mathrm{cm}^{2}$ ) substrate surface area. ${ }^{34,41}$ Since the NP fabrication process is essentially based on two simple and cost-effective steps, it opens new possibilities to tailor materials at the nanometer scale without the use of expensive lithographic tools.

Despite the aforementioned advantages, challenges in the nanofabrication procedure still remain. The SERS background noise of the Ag NP structures is considerable and the SERS enhancement factor is not optimized. These shortcomings could inhibit molecular detection at 
$<\mathrm{nM}$ concentrations. In order to develop the leaning Ag NP structures into a robust SERS platform, a more thorough understanding of the fabrication process capabilities and limitations is required. There is a need for further theoretical and experimental work to address (i) the nature of the resonant Ag NP excitations, and subsequently (ii) evaluate experimental procedures that affect the SERS intensity, uniformity and background signals.

In this work, we first provide theoretical insight into the resonant Ag NP excitations. Scattering spectra and E-field enhancement distributions around single Ag NPs were simulated using the finite element method (FEM). We find that the optical properties of $\mathrm{Ag}$ NPs are dominated by an Ag cap cavity LSPR mode. Importantly, the E-field enhancement maps show that the largest enhancement is located at the bottom of the Ag cap and in contact with the Si NP. This allows us to investigate experimental process parameters that affect the SERS signal intensity and the background noise measured from Ag NPs. Fabrication process steps for removing Si reactive ion etching (RIE) by-products from the Si NP surface that constitute the SERS background noise are introduced. We systematically expose Si NPs to $\mathrm{O}_{2}$-plasma treatment and show that the process can be utilized for both the Si NP surface cleaning and to reduce the average Ag NP cluster size. The later gradually reduces the observed SERS intensities. Cr adhesion layers on Si NPs can also be used to reduce background noise. However, the plasmon coupling losses become non negligible for $>3 \mathrm{~nm}$ Cr layers and the SERS intensity is considerably decreased. In addition, we vary the Ag NP height and the thickness of the Ag metal film to obtain maximum SERS intensities and signal uniformities. Finally, the experimentally optimized Ag NP substrates are evaluated by SERS detection at low analyte concentrations $(<\mathrm{nM})$. 


\section{THEORETICAL METHODS}

The theoretical section is aimed at helping to understand the nature of the resonant Ag NP excitations and is used as a general guideline to interpret the experimental data. Since the nanostructures are fabricated using maskless lithography, a variation in the Si NP width and Ag cap surface morphologies is inherent in the fabrication process. Therefore, the Ag NP geometry was constructed using only the experimentally observed representative Ag NP dimensions. An Ag metal film below the NPs is not included in the theoretical model. An isolated $\mathrm{Ag} \mathrm{NP}$ is modeled as a $\mathrm{Si}$ cylinder with a rounded bottom and a cone-shaped tip covered by the Ag metal film, see schematic picture in Figure 1 (a). 3D FEM is utilized to calculate scattering spectra and the electric field enhancement distribution around a single $\mathrm{Ag}$ NP. Both $\mathrm{Si}$ and $\mathrm{Ag}$ materials are modeled using experimentally obtained complex permittivities $^{43,44}$ and the surrounding medium is set to vacuum $(n=1)$. A perfectly matched layer (PML) is integrated in the model and adjusted to minimize non-physical reflections at the boundaries. A highly non-uniform adaptive mesh is used to increase the accuracy of calculation, especially near the structure's geometric singularities. The mesh is refined until the EM field converges throughout the solution domain. By solving time-harmonic Maxwell equations under different incident wavelengths, scattering spectra and the E-field enhancement distribution $\left(|E| /\left|E_{0}\right|\right)$ over the domain of interest can be calculated.

Figure 1 (b) shows the simulated scattering cross section spectra of a single Ag NP with varying Si NP radii of $r=16,20$ and $24 \mathrm{~nm}$. For $r=20 \mathrm{~nm}$, two LSPR peaks at 650 and 800 $\mathrm{nm}$ can be observed and the result is in good agreement with the experimentally obtained $\mathrm{Ag}$ NP scattering spectrum, Figure 1 (c). Due to variations in the radius of the fabricated Si NPs, the measured scattering spectrum exhibits a broader LSPR peak compared to the simulated ones. The simulated E-field enhancement distributions $\left(|E| /\left|E_{0}\right|\right)$ around the Ag NP are shown in Figure 1 (d). The E-field enhancement distribution at $650 \mathrm{~nm}$ exhibits a clear dipolar pattern, i.e. the largest E-field enhancement factor $(\mathrm{EF})$ is observed at the Ag cap edges and is 
$|E| /\left|E_{0}\right| \sim 25$. The most significant LSPR peak is at $800 \mathrm{~nm}$, and the E-field is spatially confined at the bottom of the Ag cap, i.e. the E-field enhancement originates in the Ag cap cavity, see Figure 1 (d). The maximum EF for the Ag cap cavity mode is $\sim 149$, i.e. $\sim 6$ times larger compared to the $650 \mathrm{~nm}$ mode. The resonance peak position of the cavity mode is sensitive to the radius of the Si NP, as is shown in Figure 1 (b). When the radius $r$ is increased from $16 \mathrm{~nm}$ to $24 \mathrm{~nm}$, the LSP resonance exhibits $\sim 100 \mathrm{~nm}$ red-shift.

The above results indicate some Ag NP fabrication challenges. First of all, a part of the Si NP surface is within the area of high EM fields that originate from the Ag cap cavity. Since the Si surface is covered by the Si RIE byproducts, the SERS background noise can be enhanced thus inhibiting detection of target molecules at extremely low concentrations. Process steps to control the Si NP surface contamination level are therefore necessary. Furthermore, the use of $\mathrm{Cr}$ adhesion layers for the $\mathrm{Ag}$ metal deposition can compromise the SERS signal and needs to be experimentally verified. Simulation of a NP by replacing the Ag cap cavity material with $\mathrm{Cr}$ shows that the plasmon resonance at $\sim 800 \mathrm{~nm}$ vanishes completely, see supporting information Figure S.1. Calculations also show that the size and shape of the Ag cap have minor influence on the position of the LSPR wavelength of the cavity mode, and that the largest E-field enhancement is observed at the bottom of the Ag cap for both isolated Ag NPs and nearly touching Ag NP dimers, see supporting information Figures S.4 and S.5, respectively. The results confirm that the LSPR position of the cavity mode is highly sensitive to the radius and refractive index of the Si cylinder, similar to other structures exhibiting cavity LSPR. ${ }^{45}$ For SERS applications, some inhomogeneity of the Si NP width is acceptable since combined (broad) LSPR is suitable for a wide range of laser excitation wavelengths. 

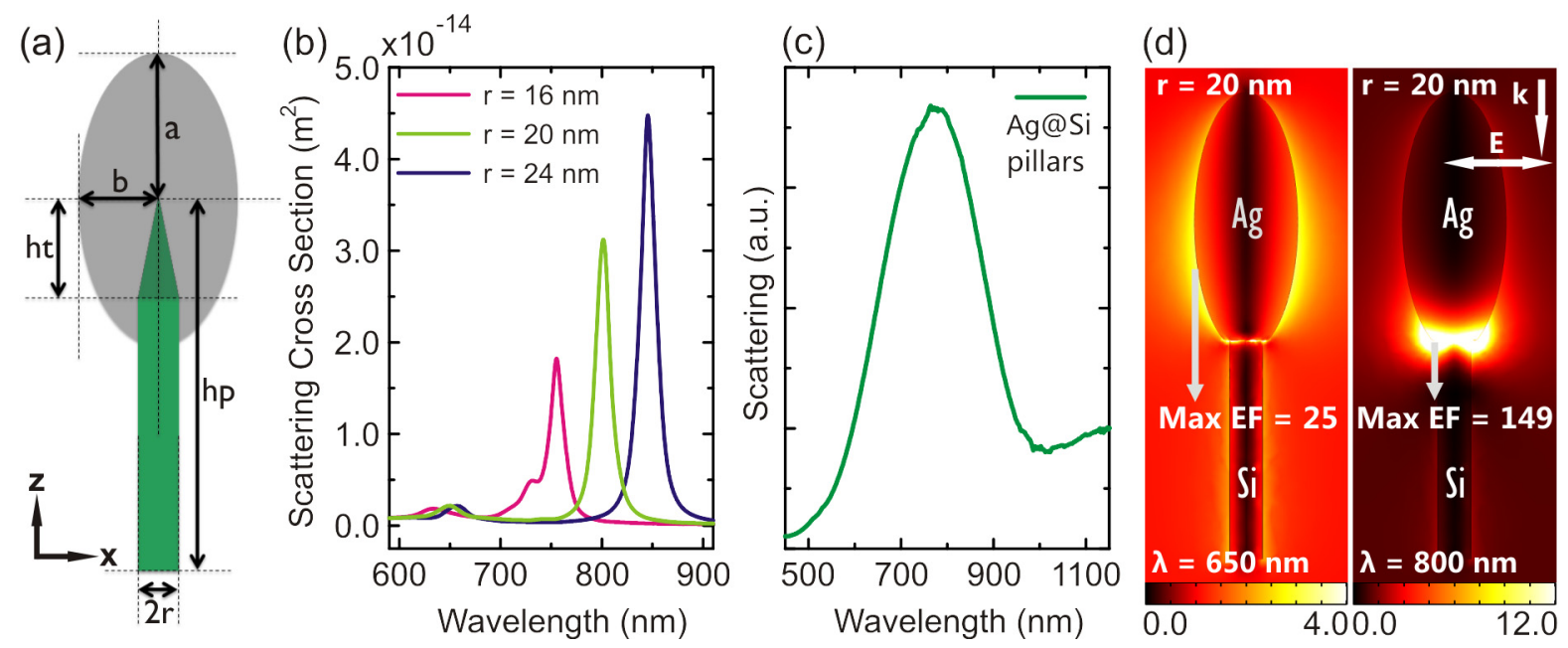

Figure 1. (a) Schematic picture of an Ag NP in the x-z plane. (b) Calculated scattering spectra of isolated Ag NPs with varying Si pillar radius, $r$. The Ag NP parameters used were: $a=155$ $\mathrm{nm}, \mathrm{b}=62 \mathrm{~nm}, \mathrm{ht}=100 \mathrm{~nm}$ and $\mathrm{hp}=400 \mathrm{~nm}$. The tips at the bottom of the Ag ellipsoid are rounded ( $5 \mathrm{~nm}$ in radius). The normal incident light is polarized along the $\mathrm{x}$-axis. The calculations were performed using FEM. (c) Measured scattering spectrum of Ag NPs with r $\approx 20 \pm 4 \mathrm{~nm}, \mathrm{hp} \approx 400 \mathrm{~nm}$ and pillar density $\rho_{\mathrm{NP}} \approx 18 \pm 2$ pillars $/ \mu \mathrm{m}^{2}$. The thickness of the ebeam evaporated $\mathrm{Ag}$ metal film is $200 \mathrm{~nm}$. (d) The calculated E-field enhancement distribution $\left(|E| /\left|E_{0}\right|\right)$ around a single Ag NP for the LSPR peaks at $\lambda=650$ and $800 \mathrm{~nm}$. The Ag NP parameters used were the same as in (b) for $r=20 \mathrm{~nm}$. The color map range for the cavity LSPR at $\lambda=800 \mathrm{~nm}$ was enhanced for clarity.

\section{EXPERIMENTAL METHODS}

The Ag NP structures were fabricated using a four-step process, schematically shown in Figure 2. First, maskless Si RIE is utilized to form Si NP structures with $r \approx 20 \pm 4 \mathrm{~nm}$ in radius and $\mathrm{h} \approx 480-1810 \mathrm{~nm}$ over an entire 4 inch Si wafer. The Si NP density in all cases is $\rho_{\mathrm{NP}} \approx 18 \pm 2$ pillars $/ \mu \mathrm{m}^{2}$. Second, an $\mathrm{O}_{2}$-plasma process is systematically applied to (i) remove Si RIE byproducts from the Si surface, and (ii) to control the Ag NP cluster size. Third, a Cr adhesion layer is evaporated onto the Si NP structure. Lastly, a Ag metal film is deposited on the Si NPs using e-beam evaporation. The deposition results in the formation of 
silver caps at the apex of the Si NPs and a silver film on the underlying Si surface. The Ag NP height, hp, in the following is defined as the distance from the middle of the $\mathrm{Ag}$ cap to the $\mathrm{Ag}$ metal surface (Fig. 1.). The relation between the Si NP height $h$, the Ag NP height hp and the thickness of $\mathrm{Ag}$ metal film $\mathrm{D}_{\mathrm{Ag}}$ is $\mathrm{h}=\mathrm{hp}+\mathrm{D}_{\mathrm{Ag}}$. Silver is chosen due to its favorable dielectric function that results in particularly strong optical resonances in the visible spectral range. The contribution to the absorption cross-section due to interband transitions is then negligible. ${ }^{46}$ Si RIE Etching: 4 inch p-type single side polished (100) wafers were used. Etching is conducted in Advanced Silicon Etcher (Surface Technology Systems MESC Multiplex ICP) at $\mathrm{SF}_{6}: \mathrm{O}_{2}$ flow ratio of 1.12 , platen power of $110 \mathrm{~W}$ and bottom chamber pressure of 36 mTorr. The Si NPs are formed at a rate of $\sim 3 \mathrm{~nm} / \mathrm{s}$.

Oxygen Plasma Treatment: Advanced Silicon Etcher (Surface Technology Systems MESC Multiplex ICP) is used at an $\mathrm{O}_{2}$ flow of $45 \mathrm{sccm}$, platen power of $20 \mathrm{~W}$, coil power of $800 \mathrm{~W}$ and bottom chamber pressure of 10 mTorr.

Electron Beam Evaporation of $\mathrm{Cr}$ and $\mathrm{Ag}$ : Alcatel SCM 600 is used at a pressure of $2 \times 10^{-6}$ mbar. Deposition rates are $3 \AA / s$ and $10 \AA /$ s for $\mathrm{Cr}$ and Ag evaporation, respectively. The deposited metal layer thickness varies by $\pm 3 \%$.

SERS measurements: All SERS experiments were conducted using a Thermo Scientific Raman DXR microscope. The signal collection time was $1 \mathrm{~s}$ and averaged 3 times, using a 25 $\mu \mathrm{m}$ slit and a $0.8 \mu \mathrm{m}$ in diameter laser spot. The microscope was coupled to a single grating spectrometer with a $5 \mathrm{~cm}^{-1}$ FWHM spectral resolution and a \pm 2 wavenumber accuracy. All SERS spectra were collected at a laser power of $0.1 \mathrm{~mW}(0.5 \mathrm{~mW}$ in Figure 3$)$, using a $10 \mathrm{x}$ objective lens and an excitation wavelength of $780 \mathrm{~nm}$ unless stated otherwise. The Ag NP SERS substrates were investigated using $10 \mathrm{mM}$ trans-1,2-bis (4-pyridyl) ethylene (BPE) dissolved in ethanol. Droplets of BPE or MiliQ water $(5 \mu 1)$ were deposited and left for drying. The droplets spread over the whole Ag NP surface area $(5 \times 5 \mathrm{~mm})$ in several seconds. The 
SERS signal mapping was performed using $1 \mathrm{mM}$ BPE, 10x objective lens, a $5 \times 5 \mathrm{~mm}$ scan area and $30 \mu \mathrm{m}$ signal collection steps.

Dark-field Scattering Measurements: The scattering signal of the Ag NPs was acquired using a dark-field microscopy system. Light from a halogen lamp (Instrument Systems, Model: DLS 500) is coupled into an optical fiber to irradiate the Ag NP sample. The incident angle was 30 degrees. The scattered light was collected using a 10x objective lens and recorded using a spectrometer (Instrument Systems, Model: Spectro 320-141). Finally, the obtained scattering signal was divided by the reference white light spectrum in order to obtain the scattering signal from Ag NPs.

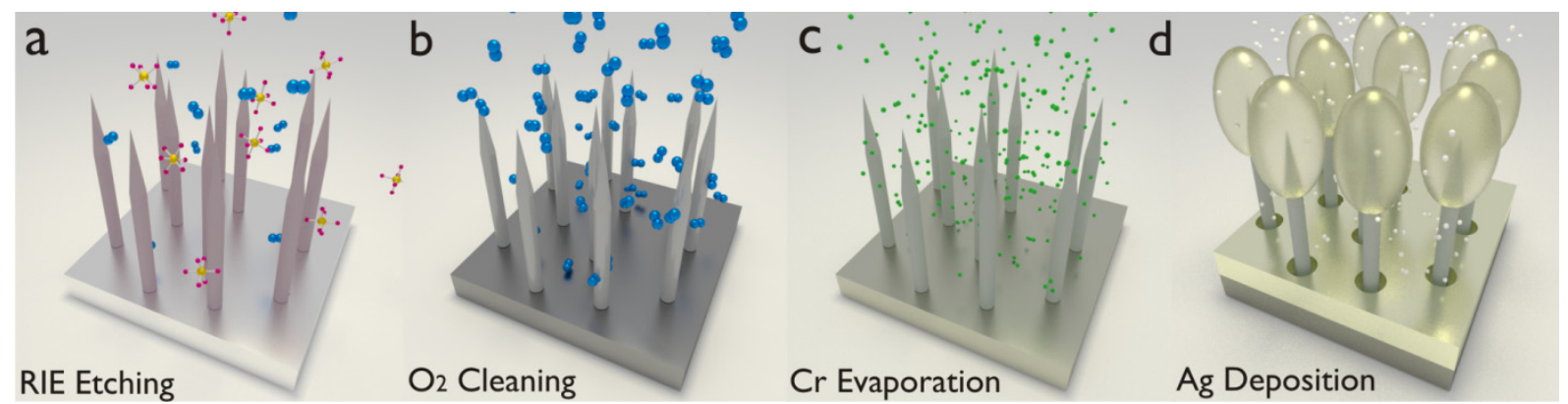

Figure 2. Summary of the fabrication process steps for the Ag NP SERS substrates. (a) Vertically standing Si pillars were produced using maskless RIE, $\mathrm{r} \approx 20 \pm 4 \mathrm{~nm}, \mathrm{~h} \approx 480-$ $1810 \mathrm{~nm}$ and pillar density $\rho_{\mathrm{NP}} \approx 18 \pm 2$ pillars $/ \mu \mathrm{m}^{2}$. (b) The Si NP were treated using $\mathrm{O}_{2}$ plasma, $\mathrm{t}=0-10$ min. (c) Deposition of the $\mathrm{Cr}$ adhesion layer, $\mathrm{D}_{\mathrm{Cr}}=0-10 \mathrm{~nm}$. (d) Evaporation of $\mathrm{Ag}$ metal film, $\mathrm{D}_{\mathrm{Ag}}=125-300 \mathrm{~nm}$. 


\section{RESULTS AND DISCUSSION}

\section{$\mathrm{O}_{2}$-plasma Treatment}

In this section we study the influence of $\mathrm{O}_{2}$-plasma exposure on the SERS signal intensity and the background noise. The Si NP structures are plasma etched using $\mathrm{SF}_{6}$ and $\mathrm{O}_{2}$ gases, and therefore Si etching byproducts are expected to cover the Si surface. To evaluate this, all substrates in this section were fabricated using identical experimental conditions yielding $\mathrm{Si}$ $\mathrm{NP}$ arrays with $\mathrm{r} \approx 20 \pm 4 \mathrm{~nm}, \mathrm{~h} \approx 600 \pm 30 \mathrm{~nm}$ and $\rho_{\mathrm{NP}} \approx 18 \pm 2$ pillars $/ \mu \mathrm{m}^{2}$. The structures were then exposed to $\mathrm{O}_{2}$-plasma for 0-10 min and covered by a $200 \mathrm{~nm}$ thick Ag film without $\mathrm{Cr}$ adhesion layers. The height of the fabricated Ag NP structures is $\mathrm{hp} \approx 400 \mathrm{~nm}$. The SERS spectra were recorded before and after exposing the samples to a $1 \mu \mathrm{L}$ BPE solution. The summarized results are presented in Figure 3.

Figure 3 (a) shows the SERS spectra from Ag NP substrates treated by $\mathrm{O}_{2}$-plasma, $\mathrm{t}=0-10 \mathrm{~min}$. The observed Raman modes at 520, 962 and $1190 \mathrm{~cm}^{-1}$ correspond to $\mathrm{Si}-\mathrm{Si}, \mathrm{SiF}_{3}{ }^{48}$ and $\mathrm{Si}-\mathrm{O}$ groups $^{53}$, respectively. Treating the samples for $1 \mathrm{~min}$ with an $\mathrm{O}_{2}$-plasma readily reduces the signal from the $\mathrm{SiF}_{3}$ group by a factor of $\sim 5$, and the $\mathrm{Si}-\mathrm{O}$ signal from the $\mathrm{SiOF}_{2}$ group ${ }^{53}$ disappears. For prolonged $\mathrm{O}_{2}$-plasma exposures $(>4 \mathrm{~min})$ the $\mathrm{SiF}_{3}$ peak vanishes, see Figure $3(\mathrm{a})$

To determine the influence of the $\mathrm{O}_{2}$-plasma step on the SERS signal from the analyte molecules, $1 \mu \mathrm{L}$ droplets of BPE were deposited and left to dry. The droplets spread over the whole Ag NP surface in several seconds regardless of the $\mathrm{O}_{2}$-plasma time. Upon solvent drying, capillary forces pull Ag NPs together causing NP clustering that increases the LSPR coupling between Ag NPs. ${ }^{41}$ The results in Figure 3(b) show that the SERS signal from BPE gradually decreases as the $\mathrm{O}_{2}$-plasma exposure time is increased. A 4 min exposure to $\mathrm{O}_{2}$ plasma reduces the SERS intensity by a factor of $\sim 4$, thus the Ag NP EF decreases dramatically. To investigate this, we examined the samples using SEM, see Figure 3(a) and (b). The SEM images reveal considerable Ag NP shape changes that affect the EF of the 
structure. In particular, the Ag NP cap is transformed into more elongated shapes. After RIE the $\mathrm{Si}$ surface is mostly populated by $\mathrm{SiF}_{\mathrm{x}}$ and $\mathrm{SiO}_{2}$ and the proportion depends on the $\mathrm{SF}_{6} / \mathrm{O}_{2}$ gas ratio. ${ }^{56}$ If the proportion of either $\mathrm{O}_{2}$ or $\mathrm{SF}_{6}$ gases in the RIE chamber is significantly increased, the $\mathrm{Si}$ surface is then dominated by $\mathrm{SiO}_{2}$ or $\mathrm{SiF}_{\mathrm{x}}$, respectively. The $\mathrm{O}_{2}$-plasma treatment gradually transforms $\mathrm{SiF}_{\mathrm{x}}$ into $\mathrm{SiO}_{2}$ which improves the $\mathrm{Ag}$ metal adhesion and consequently alters the stiffness of the Ag-coated Si NPs - limiting the cluster formation. The systematically reduced NP cluster size then leads to the decrease of the total EF, ${ }^{41}$ see SEM images in Figure 3(b).

In order to analyze the influence of the Ag cap shape changes to the cavity LSPR, we performed additional calculations (see supporting information Figure S.5). By stretching the bottom of the Ag cap by $150 \mathrm{~nm}$ along the Si pillar, and squeezing the width of the Ag cap by $40 \mathrm{~nm}$, the LSPR peak position of the cavity mode blue-shifts from $\sim 800$ to $\sim 750 \mathrm{~nm}$. Since no significant change in the E-field enhancement was observed, we attribute the reduced SERS signal intensities to changes in Ag NP cluster size. To conclude, (i) 1 min $\mathrm{O}_{2}$-plasma step removes a substantial amount of $\mathrm{SiF}_{\mathrm{x}}$ contamination while keeping the SERS signal intensity relatively unchanged, and (ii) prolonged exposures to $\mathrm{O}_{2}$-plasma reduce the EF of the structure by gradually shrinking the NP cluster sizes. 

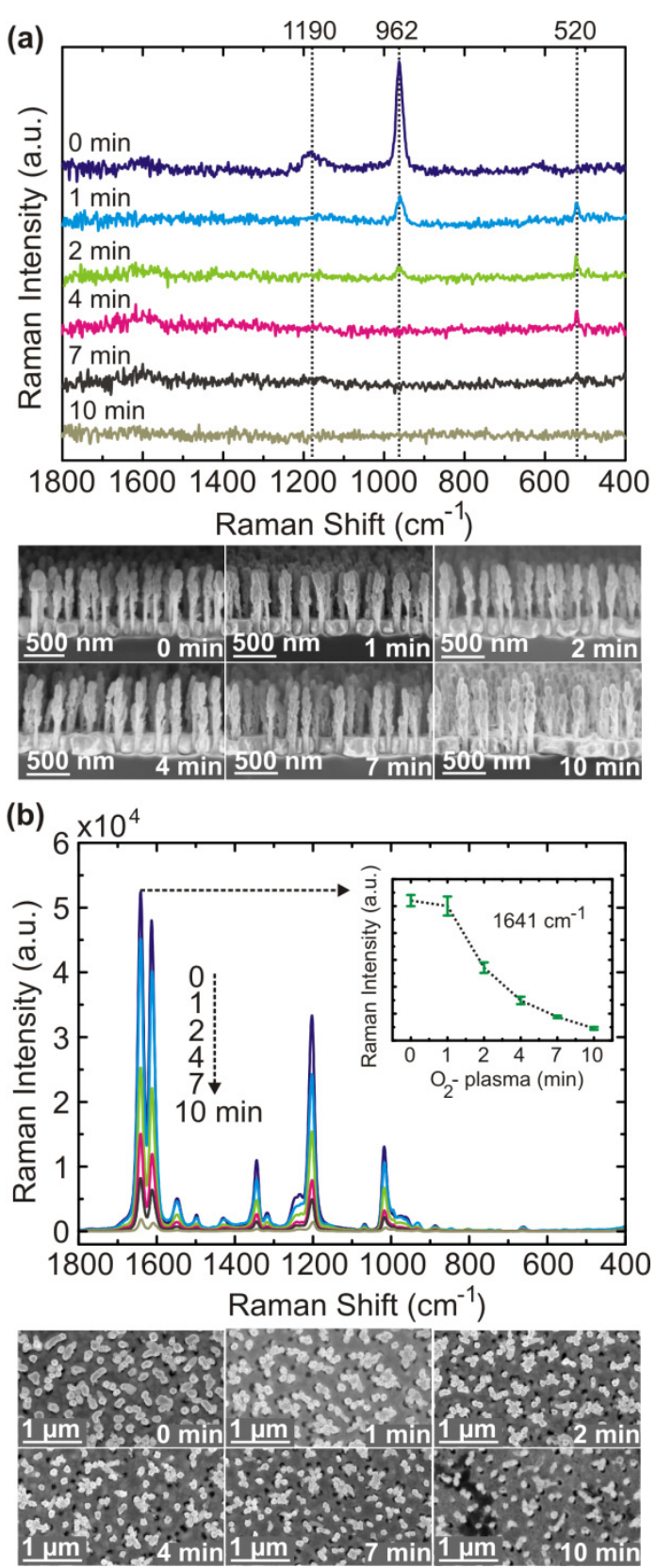

Figure 3. (a) SERS background spectra from Ag NPs after initially exposing Si NPs to an $\mathrm{O}_{2^{-}}$ plasma ( $\mathrm{t}=0$-10 min). Bottom: SEM images (side view) of the corresponding Ag NP structures. (b) SERS spectra of BPE acquired using the same Ag NP structures as shown in (a). The inset in (b) shows the signal intensity plots for the $1641 \mathrm{~cm}^{-1}$ band as a function of the $\mathrm{O}_{2}$-plasma time measured from 5 random spots. Bottom: SEM images (top view) that display the corresponding Ag NPs after exposure to BPE, where evaporation of the solvent has forced groups of individual Ag NPs into clusters (see text for details). 


\section{Cr Adhesion Layers}

$\mathrm{A} \mathrm{Cr}$ deposition step is often used to induce a more uniform growth of $\mathrm{Ag}$ or $\mathrm{Au}$ films on $\mathrm{Si}$ or $\mathrm{SiO}_{2}$ substrates. In this section, we evaluate the influence of a $\mathrm{Cr}$ adhesion layer on the $\mathrm{Ag}$ NP SERS intensity. All substrates were fabricated using the procedure described in the previous section. The $\mathrm{O}_{2}$-plasma step was not utilized in this study. A varying thickness of the Cr adhesion layer $(0-10 \mathrm{~nm})$ and a $200 \mathrm{~nm}$ thick Ag metal film were deposited using e-beam evaporation. All SERS spectra were recorded before and after exposing the Ag NP substrates to BPE.

The results are presented in Figure 4. The SERS background noise from Ag NP structures can be significantly reduced by increasing the thickness of the $\mathrm{Cr}$ layer $\left(\mathrm{D}_{\mathrm{Cr}}\right)$, shown in Figure 4(a). At $D_{C r}=6 \mathrm{~nm}$, the signal from the $962 \mathrm{~cm}^{-1}$ mode is reduced by a factor of $\sim 4$ and the response from both $\mathrm{Si}-\mathrm{O}$ and $\mathrm{SiF}_{3}$ groups is negligible at $\mathrm{D}_{\mathrm{Cr}}=10 \mathrm{~nm}$. Similarly to the $\mathrm{O}_{2^{-}}$ plasma treatment case, SERS signals from BPE decrease gradually as a function of the $\mathrm{Cr}$ layer thickness. Although, a $3 \mathrm{~nm}$ Cr layer is advantageous for reducing background noise, the effect is generally quite small for both the background and the SERS intensity from BPE. In Figure 4(b), the recorded SERS signals drop significantly for $D_{C r}>3 \mathrm{~nm}$, and for $D_{C r}=10 \mathrm{~nm}$ the SERS intensity is reduced by a factor of $\sim 1.5$. Interestingly, the SEM images in Figure 4 (a) and (b) reveal that even for thicker Cr layers, e.g. $D_{\mathrm{Cr}}=10 \mathrm{~nm}, \mathrm{Ag}$ cap shapes and stiffness properties remain similar. As expected, the SERS intensity drop is caused by plasmon coupling losses introduced through the $\mathrm{Cr}$ adhesion layer. $\mathrm{Cr}$ layers introduce loss to the coupling path of the cavity LSPR mode, thus the corresponding E-field enhancement factor is reduced. We verified these findings using FEM calculations, see Figure S.1. The results confirmed that by replacing the Ag cap cavity material with $\mathrm{Cr}$, the cavity resonance vanishes completely. Overall, the results in Figure 4 indicate that the background decreases primarily due to the reduced SERS performance of the Ag NP structures. 
Finally, applying both $\mathrm{O}_{2}$-plasma and then $\mathrm{D}_{\mathrm{Cr}}=10 \mathrm{~nm}$ reduces the SERS signal from BPE by a factor of $>20$, Figure 4 (b). The corresponding SEM image resembles findings in the previous section and further strengthens the explanation that the optical properties of Ag NP substrates are primarily altered via the $\mathrm{O}_{2}$-plasma step that induces changes in Ag cap shapes and inter-pillar leaning (Ag NP clustering). 


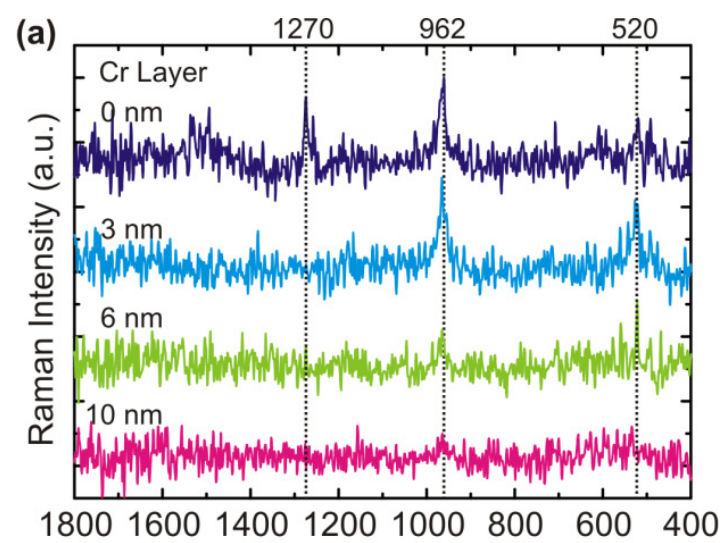

Raman Shift $\left(\mathrm{cm}^{-1}\right)$

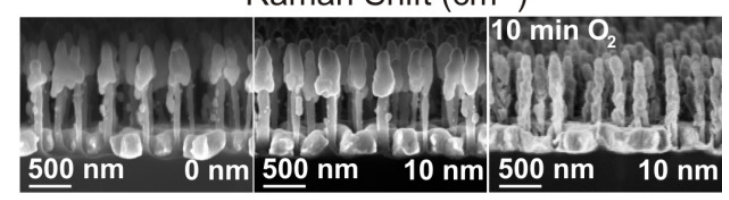

(b)
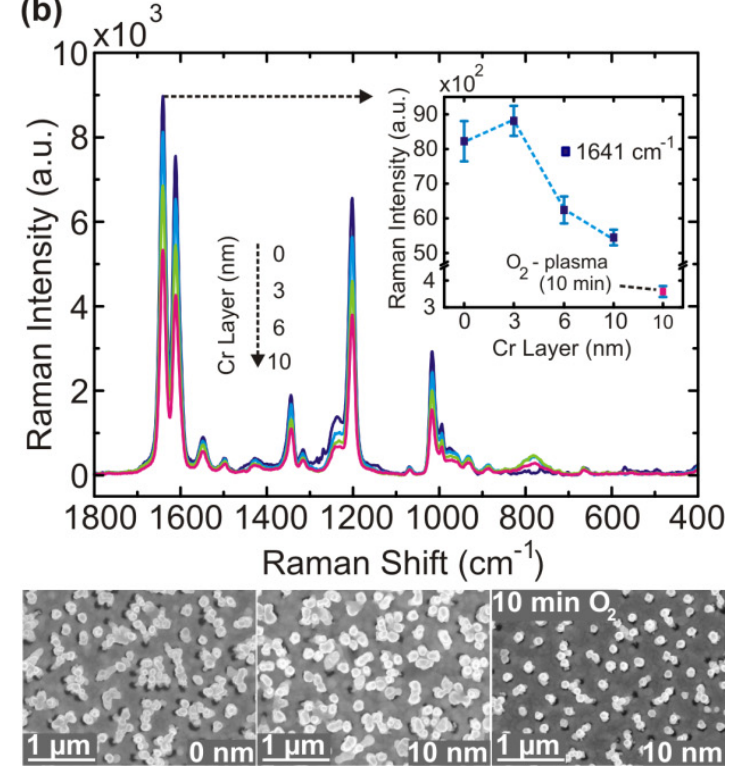

Figure 4. (a) SERS background spectra from Ag NPs structures with different Cr layer thicknesses $\left(\mathrm{D}_{\mathrm{Cr}}=0-10 \mathrm{~nm}\right)$. The $\mathrm{Cr}$ coatings were applied to the Si NP structures prior to Ag metal deposition. Bottom: SEM images (side view) for $\mathrm{D}_{\mathrm{Cr}}=0$ and $10 \mathrm{~nm}$, respectively and for using a 10 min $\mathrm{O}_{2}$-plasma prior to coating of the Si NPs with $10 \mathrm{~nm} \mathrm{Cr}$. (b) SERS spectra of BPE using the same structures shown in (a). The inset in (b) shows the signal intensity plots for the $1641 \mathrm{~cm}^{-1}$ band as a function of the Cr layer thickness measured from 5 random spots. Bottom: SEM images (top view) illustrate clustering of the Ag NPs after exposure to BPE. 


\section{Height of the Ag NPs}

In the previous sections we studied the influence of $\mathrm{O}_{2}$-plasma treatment and $\mathrm{Cr}$ adhesion layers on the SERS performance of leaning Ag NP structures. The results showed that the Ag NP cluster size plays a major role in the enhancement of the Raman signal. In this section, we study the SERS intensity changes caused by an increased Ag NP height. Si NP structures with $\mathrm{r}=20 \pm 4 \mathrm{~nm}, \rho_{\mathrm{NP}}=18 \pm 2$ pillars $/ \mu \mathrm{m}^{2}$ and varying heights $\mathrm{h} \approx 480-1810 \mathrm{~nm}$ were fabricated across an entire 4 inch Si wafer. The Si NP height was systematically increased by adjusting the Si RIE process time, see Experimental Section. All Si NP substrates were covered by a $200 \mathrm{~nm}$ thick Ag film yielding the Ag NP heights of $\mathrm{hp} \approx 280-1610 \mathrm{~nm} . \mathrm{O}_{2}$-plasma and $\mathrm{Cr}$ adhesive layer steps were not included in the fabrication process.

The SERS performance of the fabricated Ag NP structures was evaluated using BPE dissolved in ethanol. Since taller Ag NP structures form larger Ag NP clusters, the uniformity of the SERS signal can be affected. We therefore collected line series SERS spectra over a distance of $1 \mathrm{~mm}$, see data in Figure 5 (a-e). The results show that the SERS intensity is relatively stable, even though the Ag NP clusters are significantly larger for taller Ag NPs, e.g. see the SEM images for $\mathrm{hp}=1210$ and $1610 \mathrm{~nm}$. The averaged (100 spectra) SERS intensity for the $1641 \mathrm{~cm}^{-1}$ BPE band as a function of the Ag NP height is shown in Figure 5 (f). The intensity is highest for $\mathrm{hp} \approx 390 \mathrm{~nm} \mathrm{Ag} \mathrm{NPs} \mathrm{and} \mathrm{then} \mathrm{gradually} \mathrm{decreases} \mathrm{with} \mathrm{increased} \mathrm{Ag}$ NP height. Similar phenomenon can be observed using a $532 \mathrm{~nm}$ laser excitation wavelength, i.e. the largest SERS intensity is found for the $\mathrm{hp}=390 \mathrm{~nm} \mathrm{Ag} \mathrm{NPs}$. The observed signal intensity ratio for 780 vs. $532 \mathrm{~nm}$ excitation wavelengths is close to 1.5:1 (see Figure S.2). Recalling that the LSPR of the cavity mode for isolated Ag NPs is at $\lambda_{\mathrm{LSPR}}=800 \mathrm{~nm}$, the result is in a qualitative agreement with theoretical findings in Figure 1.

Interestingly, an increased Ag NP cluster size has a negative effect on the overall recorded SERS intensity. One explanation could be that BPE molecules are spread over a larger surface area using e.g. $\mathrm{hp}=1610 \mathrm{~nm}$ compared to $\mathrm{hp}=390 \mathrm{~nm} \mathrm{Ag} \mathrm{NPs}$. Since Ag NP substrates are 
hydrophilic towards ethanol, an increase in Ag NP height could lead to inhomogeneous adsorption of BPE, i.e. fewer BPE adsorb on the Ag cap surface due to fast solvent evaporation. This can lead to a different SERS intensity behavior for analytes deposited in gaseous or liquid forms. To verify this, $10 \mathrm{mM}$ thiophenol dissolved in ethanol was evaporated for $10 \mathrm{~min}$ on each of the fabricated substrates. After this, leaning of the Ag NPs was induced using $1 \mu \mathrm{L}$ ethanol droplets. Results showed that the SERS intensity behavior is similar to that observed in Figure 5 (f) (See Figure S.2).

The SERS intensity variation could be partly influenced by coupling of the Ag NP cap to the Ag film. This effect causes changes in the particle LSPR peak position as a function of the distance to the metal film. ${ }^{52}$ Optical interactions of plasmonic particles coupled to a metal film are most pronounced for particle-film separations of $d \leq 100 \mathrm{~nm}^{52} \mathrm{Hu}$ et al. have demonstrated experimentally that for isolated $60 \mathrm{~nm}$ diameter Ag particles, LSPRs can be tuned by adjusting the Au film-particle spacing. ${ }^{57}$ Noticeable shifts in the particle LSPR start to occur at $d \approx D$, where $d$ is film-particle separation distance and $D$ is particle diameter. In Figure 5 (a) and (b), the separation between the Ag cap and the Ag metal film is close to $h p \approx$ $2 a$, therefore Ag cap plasmon coupling to the Ag metal film can contribute to the observed SERS intensity variation. However, a more detailed study focused on the Ag NP optical properties is required to assess the sharp SERS intensity increase observed at hp $=390 \mathrm{~nm}$. 

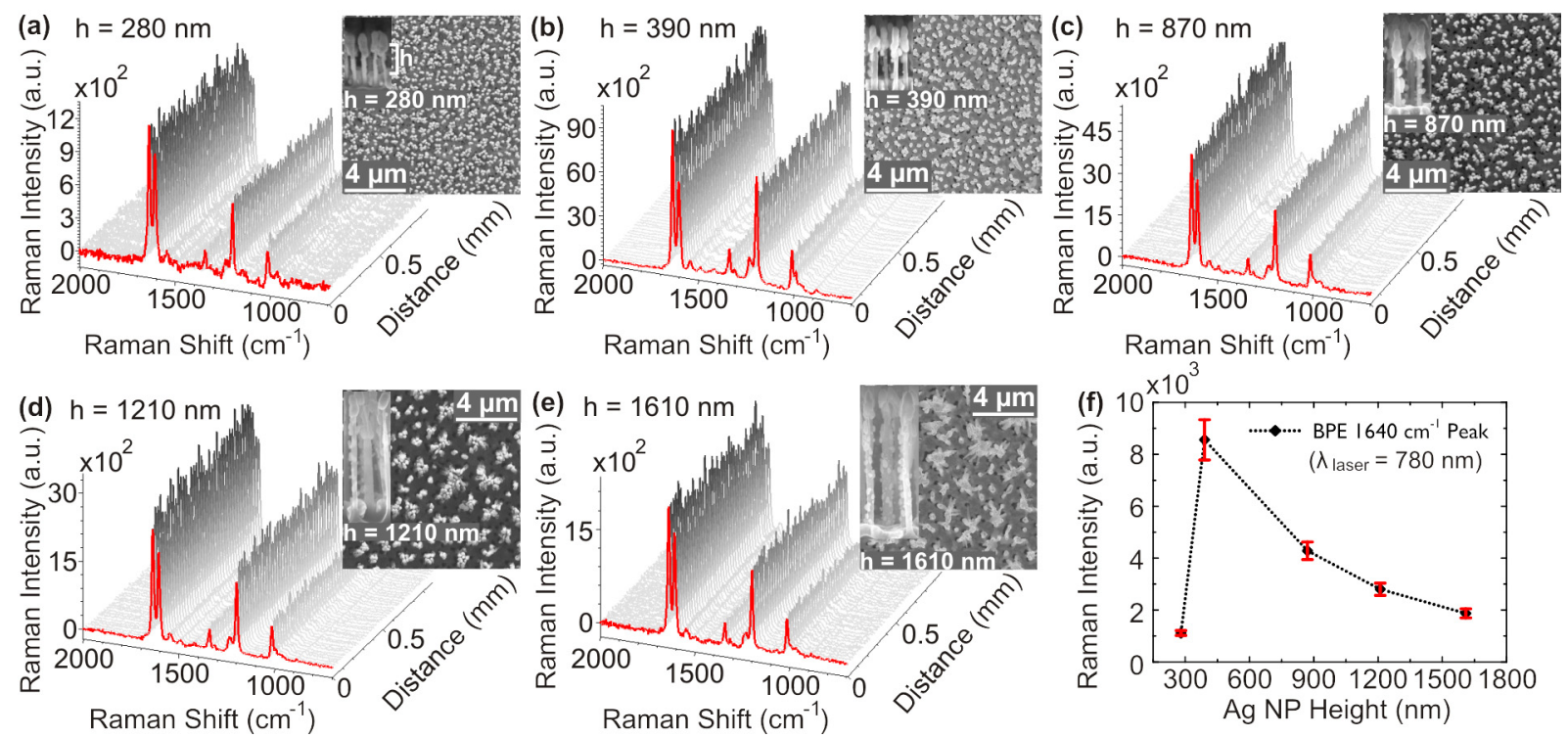

Figure 5. (a)-(e) Line series SERS spectra of BPE deposited on Ag NP structures with varying NP height $(\mathrm{hp} \approx 280-1610 \mathrm{~nm})$. The spectra were collected over a distance of $1 \mathrm{~mm}$ with $10 \mu \mathrm{m}$ steps (40 spectra out of 100 are shown). SEM images illustrate the corresponding Ag NP structures (side view) and clustering of Ag NP after exposure to BPE (top view). (f) The SERS signal intensity plots for the $1641 \mathrm{~cm}^{-1}$ band as a function of the Ag NP height averaged over 100 spectra.

\section{Thickness of the Ag Metal Film}

In this section we investigate experimentally the influence of the Ag metal thickness on the Efield enhancement properties of the Ag NP structures. The density of the fabricated Si NPs is kept at $\rho_{\mathrm{NP}} \approx 18 \pm 2$ pillars $/ \mu \mathrm{m}^{2}, \mathrm{r} \approx 20 \pm 4 \mathrm{~nm}$ and $\mathrm{h} \approx 600 \mathrm{~nm}$. The $\mathrm{O}_{2}$-plasma treatment and Cr deposition steps were not included in the Ag NP fabrication process. The thickness of the Ag film $\left(D_{\mathrm{Ag}}\right)$ was varied between 125 and $300 \mathrm{~nm}$, and SERS intensities for the $1641 \mathrm{~cm}^{-1}$ BPE band as a function of the Ag metal thickness is shown in Figure 6(a). Small intensity maxima and a sharp peak in the SERS intensity were found at $\mathrm{D}_{\mathrm{Ag}}=150 \mathrm{~nm}$ and $225 \mathrm{~nm}$, respectively. The SERS intensity for $\mathrm{D}_{\mathrm{Ag}}=225 \mathrm{~nm}$ is increased by a factor of 4 in comparison 
to the $\mathrm{D}_{\mathrm{Ag}}=125 \mathrm{~nm}$ case. The observed SERS intensity behavior was similar using both 532 and $780 \mathrm{~nm}$ laser excitation wavelengths, see Figure S.3.

To assess the origin for the sharp SERS intensity rise for $D_{A g}=225 \mathrm{~nm}$, we examine SEM images for $\mathrm{D}_{\mathrm{Ag}}=125,175,225$ and $250 \mathrm{~nm}$, see Figure 6 (b). First, the images show that increasing $\mathrm{D}_{\mathrm{Ag}}$ decreases the number of isolated Ag NPs, i.e. for $\mathrm{D}_{\mathrm{Ag}}>225 \mathrm{~nm}$ nearly all $\mathrm{Ag}$ NPs lean towards each other and become a part of large Ag NP conglomerates. For larger $\mathrm{D}_{\mathrm{Ag}}$, the surface area of the substrate increases enabling more BPE molecules to bind, enhancing the SERS intensity. Second, the shape of the Ag cap is transformed towards more elongated shapes. According to our theoretical findings, if the Ag ellipsoid dimension, $a$, is increased, the LSPR of the cavity mode blue-shifts towards the $780 \mathrm{~nm}$ excitation laser wavelength. The experimental data is in accordance with previous claims that the main contribution of the $\mathrm{Ag}$ NP EF comes from the NP cavity mode, whose resonance is close to $800 \mathrm{~nm}$, see Figure 1. 

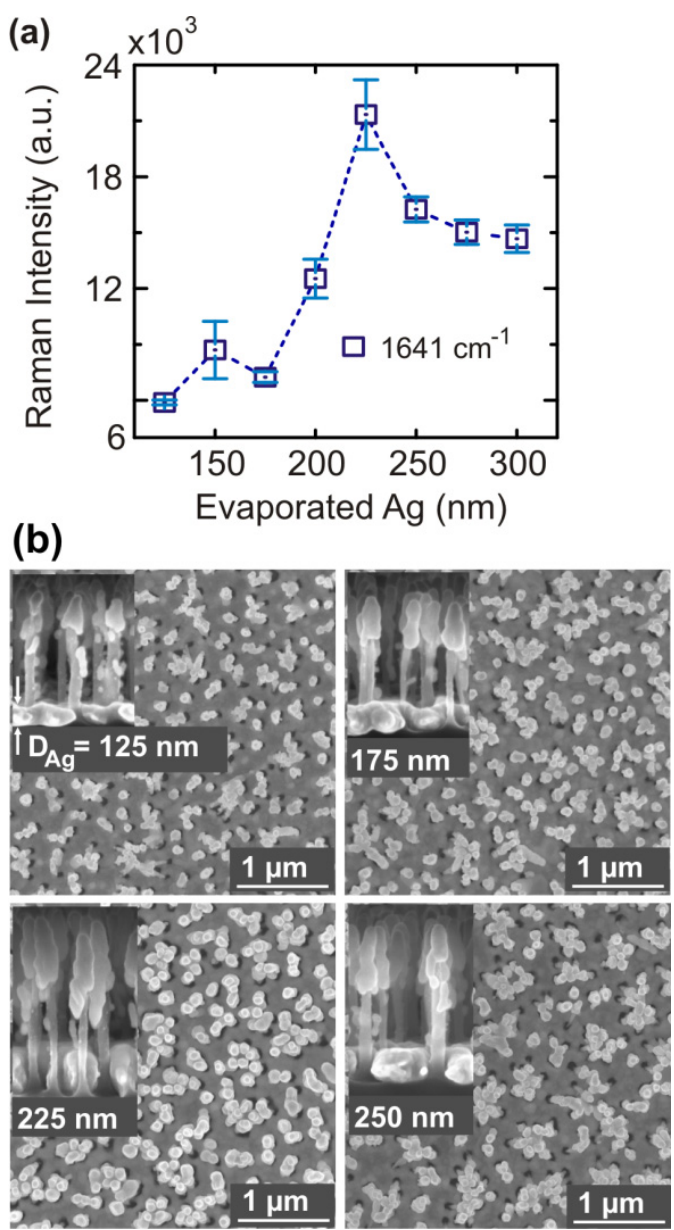

Figure 6. (a) SERS signal intensity plots for the $1641 \mathrm{~cm}^{-1} \mathrm{BPE}$ band as a function of the $\mathrm{Ag}$ layer thickness measured from 5 random spots. The Ag metal thickness was varied between $\mathrm{D}_{\mathrm{Ag}}=125$ and $300 \mathrm{~nm}$. (b) SEM images (top view) illustrate clustering of the Ag NPs after BPE deposition and solvent drying. Insets in (b) show the Ag NP structures prior to exposure to BPE.

\section{Optimized Ag NP Structures for SERS Applications}

Fianlly, all process optimizations for the Ag NP fabrication were integrated to fabricate substrates that exhibit the highest SERS intensity and uniformity over a 4" wafer. In order to verify that all experimental findings in previous sections can be consistently combined, we prepared two identical Ag NP substrate sets with $\rho_{\mathrm{NP}} \approx 18 \pm 2$ pillars $/ \mu \mathrm{m}^{2}, \mathrm{r} \approx 20 \pm 4 \mathrm{~nm}$ and $\mathrm{hp} \approx 390 \mathrm{~nm}$. The Si NP structures were then exposed to an $\mathrm{O}_{2}$-plasma for 1 and $1.5 \mathrm{~min}$, 
respectively, followed by deposition of $3 \mathrm{~nm}$ thick Cr layers. The $\mathrm{Ag}$ metal thickness $\mathrm{D}_{\mathrm{Ag}}$ was then varied between 210 and $255 \mathrm{~nm}$ for both sample sets, i.e. around the $\mathrm{D}_{\mathrm{Ag}}=225 \mathrm{~nm}$ value that yields the highest SERS intensity, see Figure 6(a).

A summary of the SERS signal intensity changes for the two sample sets is shown in Figure 7(a). Results confirm that the SERS intensity is extremely sensitive to $\mathrm{O}_{2}$-plasma treatment for all evaluated $\mathrm{Ag}$ metal thicknesses. For $\mathrm{D}_{\mathrm{Ag}}=225 \mathrm{~nm}$ an additional $30 \mathrm{~s}$ exposure to $\mathrm{O}_{2^{-}}$ plasma results in $\sim 30 \%$ decrease in the signal intensity. SEM images in Figure $7(\mathrm{~b})$ reveal that the Ag cap shape is more elongated for the $1.5 \mathrm{~min} \mathrm{O}_{2}$-plasma case. As a result, the Ag NP cluster size is reduced which translates into poorer Ag NP enhancement properties.

Results in sections 4.1-4.2 showed that a 1 min $\mathrm{O}_{2}$-plasma step and a $3 \mathrm{~nm} \mathrm{Cr}$ layer are useful for reducing SERS signals from the Si RIE induced surface contaminations, as seen in Figures 3 and 4, respectively. Indeed, the results in Figure 7(c) confirm that the aforementioned Si surface treatment steps are advantageous. Prior to collecting the SERS spectra, the Ag NP structures $\left(D_{A g}=225 \mathrm{~nm}\right)$ were pre-leaned using $1 \mu \mathrm{L}$ of DI water to induce the $\mathrm{Ag} \mathrm{NP}$ cluster formation and then the SERS signal from the Ag NP structures without analyte molecules was recorded. An identical Ag NP substrate without the $\mathrm{O}_{2}$-plasma and $\mathrm{Cr}$ deposition steps was measured for comparison (Figure 7(c)). The Raman scattering peaks at $962 \mathrm{~cm}^{-1}\left(\mathrm{SiF}_{3}\right), 1151 \mathrm{~cm}^{-1}(\mathrm{Si}-\mathrm{O}), 1640 \mathrm{~cm}^{-1}(\mathrm{C}=\mathrm{C})$ and $2080 \mathrm{~cm}^{-1}(\mathrm{Si}-\mathrm{C})^{48,} 49$ are significantly reduced in intensity. The weak Raman mode appearing at $800 \mathrm{~cm}^{-1}$ for the $\mathrm{SF}_{6} / \mathrm{O}_{2}$ plasma treated sample corresponds to the $\mathrm{SiF}$ group. ${ }^{48}$

In order to investigate the SERS signal uniformity, $1 \mu 1$ of BPE was dispersed on a surface of optimized Ag NPs, i.e. 1 min $\mathrm{O}_{2}$-plasma, $\mathrm{D}_{\mathrm{Cr}}=3 \mathrm{~nm}$ and $\mathrm{D}_{\mathrm{Ag}}=225 \mathrm{~nm}$. In Figure 7 (d), the SERS intensity map over a $5 \times 5 \mathrm{~mm}^{2}$ surface area for a $1613 \mathrm{~cm}^{-1}$ BPE vibrational mode is shown. The standard deviation for the displayed Raman scattering peak is around $14 \%$, and the recorded signal uniformity is highly reproducible over a surface area of $\sim 3.2$-inch in diameter on a 4-inch Ag NP substrate. 
The molecular detection limit of the experimentally optimized leaning Ag NP substrates was evaluated using different concentrations of BPE, see results in Figure 7 (e). Analyte concentrations down to $100 \mathrm{pM}$ can be detected displaying all five distinct BPE Raman bands at $1641 \mathrm{~cm}^{-1}, 1613 \mathrm{~cm}^{-1}, 1202 \mathrm{~cm}^{-1}$ and $1010 \mathrm{~cm}^{-1}$. Note that some BPE Raman modes split, e.g. the $1010 \mathrm{~cm}^{-1}$ peak in the $10 \mathrm{nM}$ BPE spectrum. This is likely caused by a high energy density that induces photochemistry reactions on the Ag surface leading to decomposition of the analyte molecules. ${ }^{54}$ The EF for the optimized Ag NP structures was then calculated according to

$E F=\frac{I_{S E R S} / N_{\text {SERS }}}{I_{\text {ref }} / N_{\text {ref }}}$

where $I_{S E R S}$ is the SERS intensity, $N_{S E R S}$ is the number of corresponding molecules within the laser spot, $I_{r e f}$ is the reference Raman signal intensity and $N_{r e f}$ is the number of molecules responsible for the Raman scattering signal. ${ }^{34}$ The obtained EF was $1.3 \cdot 10^{8}$, see calculation details in supporting information. 

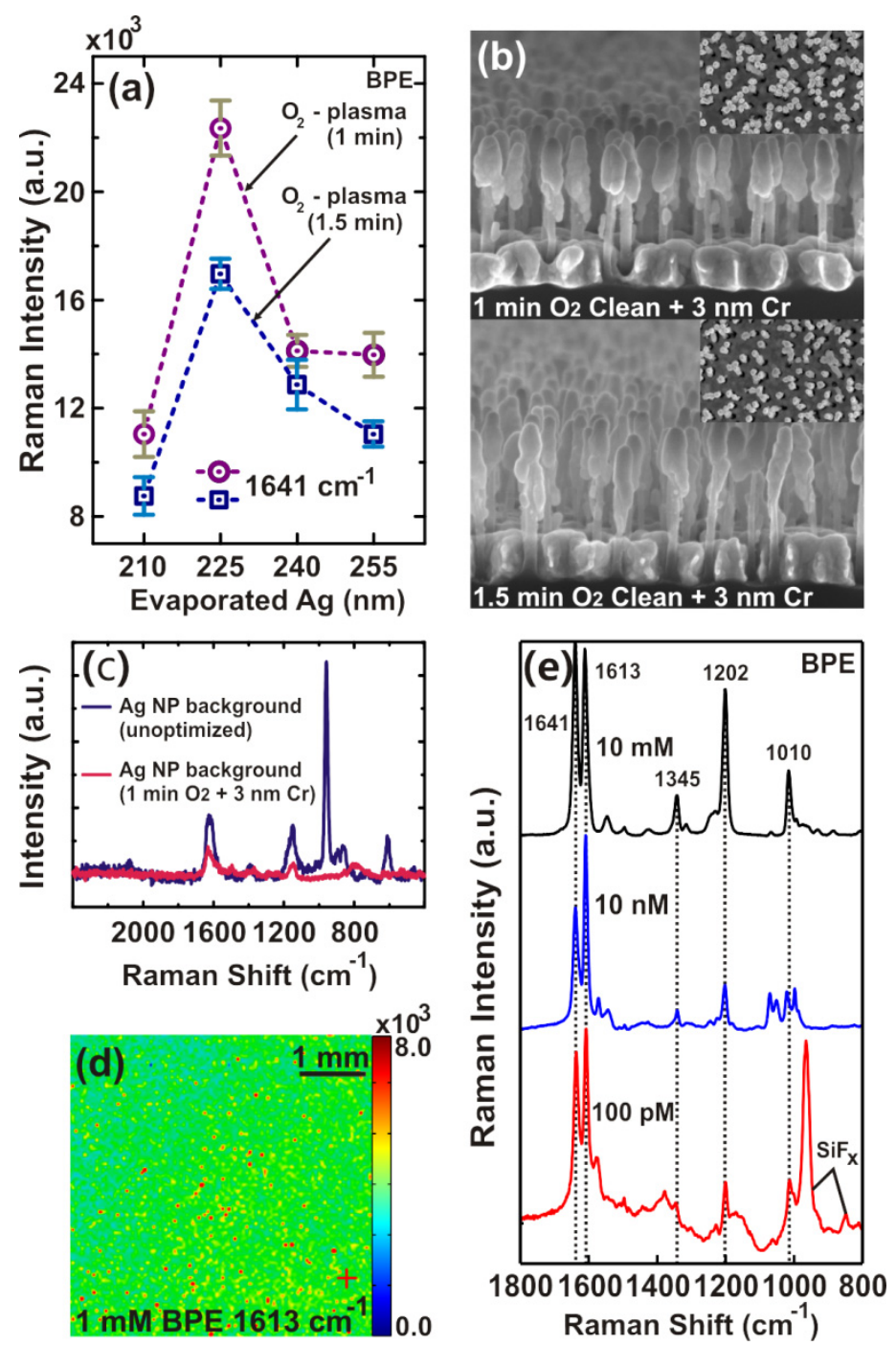

Figure 7. (a) SERS intensity plots of the $1641 \mathrm{~cm}^{-1}$ BPE mode for Ag NPs with varying Ag metal thickness. Prior to metal depositions the Si NPs were exposed to an $\mathrm{O}_{2}$-plasma for 1 and 1.5 min. (b) SEM images showing Ag NPs treated with $\mathrm{O}_{2}$-plasma, $\mathrm{D}_{\mathrm{Ag}}=225 \mathrm{~nm}$. Side and top view show the structures before and after exposure to BPE, respectively. (c) The influence of $\mathrm{O}_{2}$-plasma treatment and $\mathrm{Cr}$ layer deposition on the SERS signal from the Ag NPs. (d) Evaluation of the SERS signal uniformity using the optimized Ag NP substrate, i.e. $\mathrm{D}_{\mathrm{Ag}}=225$ $\mathrm{nm}, \mathrm{hp}=390 \mathrm{~nm}, 1 \mathrm{~min} \mathrm{O}_{2}$-plasma and $3 \mathrm{~nm} \mathrm{Cr}$. (e) SERS spectra of BPE at low concentrations using the optimized Ag NP structures. 


\section{CONCLUSIONS}

In this paper we report the fabrication steps that are important for achieving high SERS intensity and uniformity in the leaning Ag NP structures fabricated using maskless lithography. The fabrication process contains four simple, cost-effective and quick steps that are compatible with high volume manufacturing process flows: (i) Si RIE, (ii) $\mathrm{O}_{2}$-plasma treatment, (iii) deposition of the $\mathrm{Cr}$ adhesion layer, and (iv) evaporation of the Ag metal. First, the nature of the E-field enhancement in isolated Ag NPs was theoretically investigated using FEM. Calculations showed that the optical properties of single Ag NPs are dominated by a distinct Ag cap cavity resonance mode found close to $\sim 800 \mathrm{~nm}$. The resonance position can primarily be tuned via the diameter of the Si NP. The near-field plots produced at the resonance showed that the incident field is spatially confined at the Ag cap cavity, i.e. at the bottom of the Ag cap. This allowed us to attribute the E-field enhancement in Ag NPs to plasmon coupling between Ag cap cavity LSPRs that contribute most to the enhancement of the Raman signal. Moreover, since these hot spots are located at the Ag-Si interface, we established that the measured background noise from the Ag NPs corresponds to Si RIE byproducts that populate the Si NP surface.

We then showed experimentally that $\mathrm{O}_{2}$-plasma process and $\mathrm{Cr}$ adhesion layers can be used to improve the SERS signal intensity and reduce the contribution from the $\mathrm{Si}$ surface contaminants. Importantly, by controlling the $\mathrm{O}_{2}$-plasma exposure time the background noise from Ag NPs can be significantly reduced. However, a prolonged exposure to the $\mathrm{O}_{2}$-plasma induces a more uniform Ag growth on Si NPs, which produces elongated Ag cap shapes. Consequently, the Ag NP cluster size, thus SERS intensity, is systematically reduced. The Cr adhesion layer $(3 \mathrm{~nm})$ can also be utilized to further reduce the contribution from the contaminated Si NP surface. However thicker Cr layers $(>3 \mathrm{~nm})$ introduce loss which weakens the plasmon coupling in the Ag cavity. Moreover, by adjusting the Si NP height and 
thickness of the evaporated Ag film, we found that the SERS intensity can be systematically varied.

Finally, we combined all our experimental findings and presented a recipe to fabricate Ag NP structures that exhibit the highest SERS intensity. The Raman scattering signal was then recorded over a larger surface area with standard deviations of $\sim 14 \%$ across a $5 \mathrm{~mm}$ x $5 \mathrm{~mm}$ chip. The result was reproducible over a $\sim 3.2$ inch in diameter surface area. We tested the substrate performance by recording BPE spectra down to a concentration of $100 \mathrm{pM}$, displaying clear Raman vibration modes specific to BPE.

Experimental findings suggest that the Ag NP substrates are strong candidates for obtaining a reliable SERS detection of molecular species at ultra-low concentrations. We emphasize that the nanofabrication process is simple, cost-effective, CMOS compatible and could be suitable for mass-production in standard IC foundries utilizing even larger Si carrier wafers.

\section{Acknowledgement}

This work has been funded by the NAPLAS project, The Danish Council for Independent Research. K. B. Mogensen is thankful for support from The Danish National Advanced Technology Foundation (HTF, grant no. 083-2012-1). The manuscript was written through contributions of all authors. All authors have given approval to the final version of the manuscript. Authors would like to thank Prof. Mikael Käll and Stephan S. Keller for stimulating discussions and comments. The authors declare no competing financial interest.

\section{Supporting Information Description}

Supporting Information Available: Calculated absorbance spectra and E-field maps for isolated Ag NPs that show the influence of the Ag cap cavity material ( $\mathrm{Cr}$ and $\mathrm{Si}$ ) on the cavity LSPR; SERS spectra of thiophenol on Ag NPs with varying NP height; SERS signal intensity plots for the $1641 \mathrm{~cm}^{-1} \mathrm{BPE}$ band as a function of the Ag layer thickness measured 
using the $532 \mathrm{~nm}$ laser excitation wavelength; SERS spectra of thiophenol on leaned and nonleaned (isolated) Ag NPs; Calculated E-field enhancement distributions (|E $\left./\left|\mathrm{E}_{0}\right|\right)$ around a single Ag NP and a NP dimer; Calculated scattering spectra of a standard Ag NP and an Ag NP with an elongated Ag cap; Evaluation of the Raman enhancement factor (EF) for the optimized substrate. This material is available free of charge via the Internet at http://pubs.acs.org

\section{REFERENCES}

(1) Sharma, B.; Cardinal, M. F.; Kleinman, S. L.; Greeneltch, N. G.; Frontiera, R. R.;

Blaber, M. G.; Schatz, G. C.; Van Duyne, R. P. High-performance SERS Substrates: Advances and Challenges. MRS Bulletin. 2013, 38, 615-624.

(2) Le Ru, E. C.; Meyer, M.; Etchegoin, P.G. Proof of Single-Molecule Sensitivity in Surface Enhanced Raman Scattering (SERS) by Means of a Two-Analyte Technique. J. Phys. Chem. B. 2006, 110, 1944-1948.

(3) Abalde-Cela, S.; Aldeanueva-Potel, P.; Mateo-Mateo, C.; Rodríguez-Lorenzo, L.; Alvarez-Puebla, R. A.; Liz-Marzán, L. M. Surface-enhanced Raman Scattering Biomedical Applications of Plasmonic Colloidal Particles. J. R. Soc. Interface. 2010, 7, S435-S450.

(4) Hutter, E.; Fendler, J. H. Exploitation of Localized Surface Plasmon Resonance. Adv. Mater. 2004, 16, 1685-1706.

(5) Willets, K. A.; Van Duyne, R. P. Localized Surface Plasmon Resonance Spectroscopy and Sensing. Annu. Rev. Phys. Chem. 2007, 58, 267-297.

(6) Mayer, K. M.; Hafner, J. H. Localized Surface Plasmon Resonance Sensors. Chem. Rev. 2011, 111, 3828-3857. 
(7) Xu, H.; Aizpurua, J.; Käll, M.; Apell, P. Electromagnetic Contributions to Singlemolecule Sensitivity in Surface-enhanced Raman Scattering. Phys. Rev. E 2000, 62, 43184324.

(8) Ye, X.; Zheng, C.; Chen, J.; Gao, Y.; Murray, C. B. Seeded Growth of Monodisperse Gold Nanorods Using Bromide-Free Surfactant Mixtures. Nano Lett. 2013, 13, 2163-2171.

(9) Rycenga, M.; Xia, X.; Moran, C. H.; Zhou, F.; Qin, D.; Li, Z.-Y.; Xia, Y. Generation of Hot Spots with Silver Nanocubes for Single-Molecule Detection by Surface-Enhanced Raman Scattering. Angew. Chem. Int. Ed. 2011, 50, 5473-5477.

(10) Khoury, C. G.; Vo-Dinh, T. Gold Nanostars for Surface-Enhanced Raman Scattering: Synthesis, Characterization and Optimization. J. Phys. Chem. C. 2008, 112, 18849-18859.

(11) Bastús, N. G.; Comenge, J.; Puntes, V. C. Kinetically Controlled Seeded Growth Synthesis of Citrate-Stabilized Gold Nanoparticles of up to $200 \mathrm{~nm}$ : Size Focusing versus Ostwald Ripening. Langmuir. 2011, 27, 11098-11105.

(12) Zhang, J.; Gao, Y.; Alvarez-Puebla, R. A.; Buriak, J. M.; Fenniri, H. Synthesis and SERS Properties of Nanocrystalline Gold Octahedra Generated from Thermal Decomposition of $\mathrm{HAuCl}_{4}$ in Block Copolymers. Adv. Mater. 2006, 18, 3233-3237.

(13) Wang, H.; Levin, C. S.; Halas, N. J. Nanosphere Arrays with Controlled Sub-10-nm Gaps as Surface-Enhanced Raman Spectroscopy Substrates. J. Am. Chem. Soc. 2005, 127, 14992-14993.

(14) Langille, M. R.; Personick, M. L.; Zhang, J.; Mirkin, C. A. Defining Rules for the Shape Evolution of Gold Nanoparticles. J. Am. Chem. Soc. 2012, 134, 14542-14554.

(15) Lim, D.-K.; Jeon, K.-S.; Hwang, J.-H.; Kim, H.; Kwon, S.; Suh, Y. D.; Nam, J.-M. Highly Uniform and Reproducible Surface-enhanced Raman Scattering from DNA-tailorable Nanoparticles with 1-nm Interior Gap. Nat. Nanotech. 2011, 6, 452-460. 
(16) Kühler, P.; Roller, E.-M.; Schreiber, R.; Liedl, T.; Lohmüller, T.; Feldmann, J. Plasmonic DNA-Origami Nanoantennas for Surface-Enhanced Raman Spectroscopy. Nano Lett. 2014, 14, 2914-2919.

(17) He, L.; Lin, M.; Li, H.; Kim, N.-J. Surface-enhanced Raman Spectroscopy Coupled with Dendritic Silver Nanosubstrate for Detection of Restricted Antibiotics. J. Raman Spectrosc. 2010, 41, 739-744.

(18) Lim, D.-K.; Jeon, K.-S.; Kim, H. M.; Nam, J.-M.; Suh, Y. D. Nanogap-engineerable Raman-active Nanodumbbells for Single-molecule Detection. Nat. Mater. 2010, 9, 60-67.

(19) Le Ru, E. C.; Etchegoin, P. G. Single-Molecule Surface-Enhanced Raman Spectroscopy. Annu. Rev. Phys. Chem. 2012, 63, 65-87.

(20) Xu, H.; Bjerneld, E. J.; Käll, M.; Borjesson, L. Spectroscopy of Single Hemoglobin Molecules by Surface Enhanced Raman Scattering. Phys. Rev. Lett. 1999, 83, 4357-4360.

(21) Betz, J. F.; Yu, W. W.; Cheng, Y.; White, I. M.; Rubloff, G. W. Simple SERS Substrates: Powerful, Portable, and Full of Potential. Phys. Chem. Chem. Phys. 2014, 16, 2224-2239.

(22) Zhang, X.; Yonzon, C. R.; Van Duyne, R. P. Nanosphere Lithography Fabricated Plasmonic Material and their Applications. J. Mater. Res. 2006, 21, 1083-1092.

(23) Abu Hatab, N. A.; Oran, J. M.; Sepaniak, M. J. Surface-Enhanced Raman Spectroscopy Substrates Created via Electron Beam Lithography and Nanotransfer Printing. ACS Nano. 2008, 2, 377-385.

(24) Sánchez-Iglesias, A.; Aldeanueva-Potel, P.; Ni, W.; Pérez-Juste, J.; Pastoriza-Santos, I.; Alvarez-Puebla, R. A.; Mbenkum, B. N.; Liz-Marzán, L. M. Chemical Seeded Growth of Ag Nanoparticle Arrays and their Application as Reproducible SERS Substrates. Nano Today. 2010, 5, 21-27. 
(25) Greeneltch, N.; Blaber, M.; Henry, A-I.; Schatz, G.; Van Duyne, R. P. Immobilized Nanorod Assemblies: Fabrication and Understanding of Large Area Surface-enhanced Raman Spectroscopy Substrates. Anal. Chem. 2013, 85, 2297-2303.

(26) Oh, Y.-J.; Jeong, K.-H. Glass Nanopillar Arrays with Nanogap-Rich Silver Nanoislands for Highly Intense Surface Enhanced Raman Scattering. Adv. Mater. 2012, 24, 2234-2237.

(27) Le Ru, E. C.; Etchegoin, P. Principles of Surface Enhanced Raman Spectroscopy and related Plasmonic Effects. Elsevier: Amsterdam, Netherland, 2008.

(28) Shumaker-Parry, J. S.; Rochholz, H.; Kreiter, M. Fabrication of Crescent-Shaped Optical Antennas. Adv.Mater. 2005, 17, 2131-2134.

(29) Wu, L. Y.; Ross, B. M.; Lee, L. P. Optical Properties of the Crescent-shaped Nanohole Antenna. Nano Lett. 2009, 9, 1956-1961.

(30) Dinish, U. S.; Yaw, F. C.; Agarwal, A.; Olivo, M. Development of Highly Reproducible Nanogap SERS Substrates: Comparative Performance Analysis and its Application for Glucose Sensing. Biosens. Bioelectron. 2011, 26, 1987-1992.

(31) Im, H.; Bantz, K. C.; Lindquist, N. C.; Haynes, C. L.; Oh, S.-H. Vertically Oriented Sub-10-nm Plasmonic Nanogap Arrays. Nano lett. 2010, 10, 2231-2236.

(32) Caldwell, J. D.; Glembocki, O.; Bezares, F. J.; Bassim, N. D.; Rendell, R. W.; Feygelson, M.; Ukaegbu, M.; Kasica, R.; Shirey, L.; Hosten, C. Plasmonic Nanopillar Arrays for Large-Area, High-Enhancement Surface-Enhanced Raman Scattering Sensors. ACS Nano. 2011, 5, 4046-4055.

(33) Ruan, C.; Eres, G.; Wang, W.; Zhang, Z.; Gu, B. Controlled Fabrication of Nanopillar Arrays as Active Substrates for Surface-enhanced Raman Spectroscopy. Langmuir. 2007, 23, $5757-5760$ 
(34) Hu, M.; Ou, F. S.; Wu, W.; Naumov, I.; Li, X.; Bratkovsky, A. M.; Williams, R. S.; Li,

Z. Gold Nanofingers for Molecule Trapping and Detection. J. Am. Chem. Soc. 2010, 132, $12820-12822$.

(35) Huang, Z.; Meng, G.; Huang, Q.; Yang, Y.; Zhu, C.; Tang, C. Improved SERS Performance from Au Nanopillar Arrays by Abridging the Pillar Tip Spacing by Ag Sputtering. Adv. Mater. 2010, 22, 4136-4139.

(36) Mao, H.; Wu, W.; She, D.; Sun, G.; Lv, P.; Xu, J. Microfluidic Surface-Enhanced Raman Scattering Sensors Based on Nanopillar Forests Realized by an Oxygen-PlasmaStripping-of-Photoresist Technique. Small. 2014, 10, 127-134.

(37) Gartia, M. R.; Xu, Z.; Behymer, E.; Nguyen, H.; Britten, J. A.; Larson, C.; Miles, R.; Bora, M.; Chang, A. S.-P.; Bond, T. C.; Liu, G. L. Rigorous Surface Enhanced Raman Spectral Characterization of Large-area High-uniformity Silver-coated Tapered Silica Nanopillar Arrays. Nanotechnology. 2010, 21, 395701.

(38) Bora, M.; Fasenfest, B. J.; Behymer, E. M.; Chang, A. S.-P.; Nguyen, H. T.; Britten, J. A.; Larson, C. C.; Chan, J. W.; Miles, R. R.; Bond, T. C. Plasmon Resonant Cavities in Vertical Nanowire Arrays. Nano Lett. 2010, 10, 2832-2837.

(39) Dawson, P.; Duenas, J. A.; Boyle, M. G.; Doherty, M. D.; Bell, S. E. J.; Kern, A. M.; Martin, O. J. F.; Teh, A.-S.; Teo, K. B. K.; Milne, W. I. Combined Antenna and Localized Plasmon Resonance in Raman Scattering from Random Arrays of Silver-Coated, Vertically Aligned Multiwalled Carbon Nanotubes. Nano Lett. 2011, 11, 365-371.

(40) Yang, X.; Ileri, N.; Larson, C. C.; Carlson, T. C.; Britten, J. A.; Chang, A. S. P.; Gu, C.; Bond, T. C. Nanopillar Array on a Fiber Facet for Highly Sensitive Surface-enhanced Raman Scattering. Optics Express. 2012, 20, 24819-24826.

(41) Schmidt, M. S.; Hübner, J.; Boisen, A. Large Area Fabrication of Leaning Silicon Nanopillars for Surface Enhanced Raman Spectroscopy. Adv. Mater. 2012, 24, OP11-OP18. 
(42) Yang, J.; Palla, M.; Bosco, F. G.; Rindzevicius, T.; Alstrøm, T. S.; Schmidt, M. S.; Boison, A.; Ju, J.; Lin, Q. Surface-Enhanced Raman Spectroscopy Based Quantitative Bioassay on Aptamer-Functionalized Nanopillars Using Large-Area Raman Mapping. ACS Nano. 2013, 7, 5350-5359.

(43) Johnson, P. B.; Christy, R. W. Optical Constants of the Noble Metals. Phys. Rev. B. 1972, 6, 4370-4379.

(44) Bass, M.; DeCusatis, C.; Enoch, J.; Lakshminarayanan, V.; Li, G.; MacDonald, C.; Mahajan, V.; Van Stryland, E. Handbook of Optics, 3rd Edition. McGraw-Hill Professional: New York, U.S., 2009.

(45) Wu, K.; Cheng, X.; Lee, L. P. Intra-particle Coupling and Plasmon Tuning of Multilayer Au/dielectric/Au Nanocrescents Adhered to a Dielectric Cylinder. Nanotechnology, 2012, 23, 055201 .

(46) Cardinal, M. F.; Rodríguez-González, B.; Alvarez-Puebla, R. A.; Pérez-Juste, J.; LizMarzán, L. M. Modulation of Localized Surface Plasmons and SERS Response in Gold Dumbbells through Silver Coating. J. Phys. Chem. C. 2010, 114, 10417-10423.

(47) Weissleder, R. A Clearer Vision for In vivo Imaging. Nature Biotechnology. 2001, 19, $316-317$

(48) Colthup, N. B.; Daly, L. H.; Wiberley, S. E. Introduction to Infrared and Raman Spectroscopy, 3rd Edition. Academic Press: San Diego, U.S., 1990.

(49) Lin-Vien, D.; Colthup, N. B.; Fateley, W. G.; Grasselli, J. G. The Handbook of Infrared and Raman Characteristic Frequencies of Organic Molecules. Academic Press: Boston, U.S., 1991.

(50) Pauling, L. C. The Nature of the Chemical Bond and the Structure of Molecules and Crystals: An Introduction to Modern Structural Chemistry. Cornell University Press: New York, U.S., 1960. 
(51) Lee, K. G.; Ahn, K. J.; Kihm, H. W.; Ahn, J. S.; Kim, T. K.; Hong, S.; Kim, Z. H.; Kim, D. S. Surface Plasmon Polariton Detection Discriminating the Polarization Reversal Image Dipole effects. Optics Express. 2008, 16, 10641-10649.

(52) Lévêque, G.; Martin, O. J. Optical Interactions in a Plasmonic Particle Coupled to a Metallic Film. Optics Express, 2006, 14, 9971-9981.

(53) Reiche, M.; Wiegand, M.; Gösele, U. Infrared Spectroscopic Analysis of Plasmatreated Si(100)-surfaces. Microchimica Acta. 2000, 133, 35-43.

(54) Alaverdyan, Y.; Johansson, P.; Käll, M. Photo-induced Transformations in 2,2':5',2'’Terthiophene Thin Films on Silver. Phys. Chem. Chem. Phys. 2006, 8, 1445-1450.

(55) Hu, Y. S.; Jeon, J.; Seok, T. J.; Lee, S.; Hafner, J. H.; Drezek, R. A.; Choo, H. Enhanced Raman Scattering from Nanoparticle-Decorated Nanocone Substrates: A Practical Approach to Harness In-Plane Excitation. ACS Nano. 2010, 4, 5721-5730.

(56) d'Agostino, R.; Flamm, D. L. Plasma Etching of $\mathrm{Si}$ and $\mathrm{SiO}_{2}$ in $\mathrm{SF}_{6}-\mathrm{O}_{2}$ Mixtures. $J$. Appl. Phys. 1981, 52, 162-167.

(57) Hu, M.; Ghoshal, A.; Marquez, M.; Kik, P. G. Single Particle Spectroscopy Study of Metal-Film-Induced Tuning of Silver Nanoparticle Plasmon Resonances. J. Phys. Chem. C. 2010, 114, 7509-7515. 
TABLE OF CONTENT IMAGE

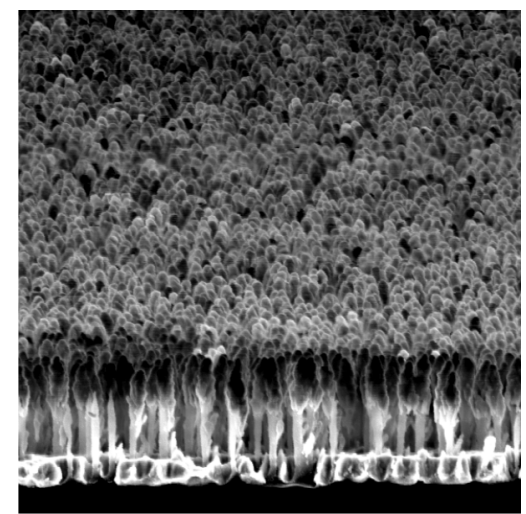

